\title{
Estimation of the lunar reflectance by ground-based observation using a tunable liquid-crystal filter telescope
}

\author{
Kazuto Saiki $^{1}$, Kimiko Saito ${ }^{1}$, Hideaki Okuno ${ }^{1}$, Akiko Suzuki ${ }^{1}$, Yuta Yamanoi ${ }^{1}$, \\ Naru Hirata ${ }^{2}$, and Ryosuke Nakamura ${ }^{3}$ \\ ${ }^{1}$ Department of Earth and Space Science, Osaka University, Toyonaka City, Osaka, Japan \\ ${ }^{2}$ Department of Computer Software, The University of Aizu, Aizu-Wakamatsu City, Fukushima, Japan \\ ${ }^{3}$ Grid Technology Research Center, Advanced Industrial Science and Technology (AIST), Tsukuba, Japan
}

(Received April 24, 2007; Revised November 20, 2007; Accepted December 11, 2007; Online published April 9, 2008)

\begin{abstract}
Lunar reflectance data are useful not only for lithological identification of the lunar surface but also for radiometric calibration and determination of exposure time for optical sensors of lunar probes. To gain data on lunar reflectance, we acquired multi-band images (five bands: 650, 750, 900, 950, and $1000 \mathrm{~nm}$ ) of the lunar surface and those of some standard stars using a liquid-crystal tunable filter (LCTF) telescope located on the peak of Mt. Haleakala (Hawaii, USA). The data obtained indicate that the reflectance data of Clementine UV/VIS is too high and that the correction factor is $0.59 \pm 0.06$ at $950 \mathrm{~nm}$. Our new reflectance data are available to the public at the web site of one of authors (K.S.). We report here our method of deriving the lunar reflectance images from the ground-based observation with a hyperspectral telescope for the users of our reflectance data. The results suggest that ground-based observation is more suitable for the radiometric calibration of the sensor of a lunar probe than laboratory data.
\end{abstract}

Key words: Clementine, the Moon, hyper-spectral telescope, reflectance, SELENE.

\section{Introduction}

The Japanese lunar probe SELENE (KAGUYA) was launched on 14 September 2007 by JAXA (Japan Aerospace Exploration Agency). As co-investigators of the SELENE project, some of the authors of this article are observing the global mineral distribution of the lunar surface in nine band images with the Lunar Imager and Spectrometer (LISM) onboard SELENE. The lunar reflectance is an important factor for determining the exposure-timesetting plan of these sensors. McEwen (1996) derived the global visible reflectance model with the images of Clementine Spacecraft launched in 1994. The UV/VIS data set and its calibration method are freely available to the public, therefore many studies have been carried out based on these data. Shkuratov et al. (2001), however, concluded that the albedo of the lunar surface determined by Clementine turns out to be a factor of 2 or 3 higher than that inferred from ground-based measurements due to the photometrically non-representative calibration standards. By comparing Clementine observations with prior ground-based observations on 15 sites on the Moon, Hillier et al. (1999) determined a good absolute calibration of the Clementine UV/VIS camera. A correction factor of 0.532 was determined as the conversion factor for converting the web site reflectances to absolute values. The latest photometric models were reported by the Robotic Lunar Observatory (ROLO) project (Kieffer and Stone, 2005). This model

Copyright (c) The Society of Geomagnetism and Earth, Planetary and Space Sciences (SGEPSS); The Seismological Society of Japan; The Volcanological Society of Japan; The Geodetic Society of Japan; The Japanese Society for Planetary Sciences; TERRAPUB. is not freely available for scientific study because the raw data have not been made accessible to the public. Our reflectance model and the calibration procedure reported here are freely available to the public, and the raw data and calibration filter tool can also be downloaded from the WEB site of one of the authors; "Moon Base Osaka (http://astrosis.ess.sci.osaka-u.ac.jp/MBO.html)".

\section{Method}

To gain lunar absolute radiance and reflectance, we acquired multi-band images of the lunar surface and those of some standard stars using a hyper-spectral telescope located on the peak of Mt. Haleakala (Hawaii, USA). Our hyper-spectral telescope system consists of a telescope, a cooling CCD camera, a liquid-crystal tunable filter (LCTF), and a notebook computer for importing images and tuning the wavelength of LCTF. The telescope is a Vixen GPED80SM with an ED (extra-low dispersion glass) apochromatic objective refractor. The effective aperture is $80 \mathrm{~mm}$ and the focal length is $720 \mathrm{~mm}$. The CCD camera is an Apogee U260 with a CCD Kodak KAF-0261E. The pixels are arranged in a $512(\mathrm{H}) \times 512(\mathrm{~V})$ array, and the pixel size is $20 \mu \mathrm{m}$. The LCTF, model VeriSpecTM NIR-07 (Cambridge Research Instrumentation), is placed between the telescope and the CCD camera. The LCTF is essentially a multistage Lyot-Ohman type polarization interference filter with an added liquid-crystal waveplate in each stage providing an electronically controllable variable retardance (Gat, 2003). The controller of the LCTF has an USB peripheral connector for direct interface to the notebook computer's USB. The filter functions like a high-quality interference 
Table 1. Observation of standard stars.

\begin{tabular}{|c|c|c|c|c|c|c|}
\hline \multirow[t]{2}{*}{ Date } & \multirow[t]{2}{*}{ Name of standard star } & \multicolumn{5}{|c|}{ Number of measurements } \\
\hline & & $650 \mathrm{~nm}$ & $750 \mathrm{~nm}$ & $900 \mathrm{~nm}$ & $950 \mathrm{~nm}$ & $1000 \mathrm{~nm}$ \\
\hline 18 Aug. 2005 & Nunki ( $\sigma$ Sagittarii) & 15 & 15 & 15 & 15 & 15 \\
\hline 20 Aug. 2005 & Fomalhaut ( $\alpha$ Piscis Austrini) & 25 & 25 & 25 & 25 & 25 \\
\hline 15 Dec. 2005 & Elnath ( $\beta$ Tauri) & 40 & 40 & 40 & 40 & 21 \\
\hline
\end{tabular}

filter, but the wavelength of light it transmits is electronically tunable and allows for the rapid, vibrationless selection of any wavelength in the near-infrared (NIR) region. The filter covers the wavelength range of 650-1100 nm with the full-width half-maximum (FWHM) of $7 \mathrm{~nm}$. The telescope is set on the equatorial mount with a tripod. It can be aimed at a celestial object and guided by an electric motor drive controlled by an external telescope computer, Vixen Skysensor 2000PC, to follow the object across the sky. The location of the observation was Science City at the peak of Mt. Haleakala, Maui, Hawaii, USA. For the calculation of lunar and stellar position, we used a longitude of $156^{\circ} 15^{\prime} 23^{\prime \prime} \mathrm{W}$, latitude of $20^{\circ} 42^{\prime} 27^{\prime \prime} \mathrm{N}$, and altitude of 3040 $\mathrm{m}$ as the location of observation. The dates of the observations were August 15-26, 2005 and December 12-18, 2005. Among all data, the data of 18 and 20 August and 15 December were selected because atmospheric conditions were relatively stable during these observational times, and the indispensable data sets that included one standard star situated close to the Moon, Vega, and the Moon were successfully obtained. The images of some standard stars, Vega, and the Moon at five bands-650, 750, 900, 950, and $1000 \mathrm{~nm}$-were obtained. Four bands among these have the same wavelength as the UV/VIS sensor of Clementine and the MI sensor of SELENE. The optical depth of the day was obtained by observing Nunki ( $\sigma$ Sagittarii), Fomalhaut ( $\alpha$ Piscis Austrini), and Elnath ( $\beta$ Tauri) as a standard star (Table 1).

\section{Data Analysis}

Dark-field and flat-field corrections were applied to each image obtained by CCD. Sky flats were used as the flat field. Atmospheric correction is indispensable to gain lunar extra-atmospheric irradiance, as aerosol, nitrogen, oxygen, and atmospheric water vapor scatter and absorb the lunar light. Atmospheric correction removes these atmospheric effects. The observed intensity $\left(D N_{\text {obs }}\right)$ of an astronomical object is expressed by the Lambert-Beer law as

$$
\ln D N_{\mathrm{obs}}=-\tau \alpha+\ln D N_{0}
$$

where $D N_{0}$ is the extra-atmospheric intensity, $\tau$ is the optical depth of the atmosphere, and $\alpha$ is the optical airmass factor expressed by elevation angle of the object; $\theta$ as $\alpha=1 / \sin \theta \cdot \ln D N_{\text {obs }}$ is linearly related to $\alpha$ as long as $\tau$ is constant. Figure 1 is an example of a Langley plot (Shiobara et al., 1996; Langley, 1881) of some $D N_{\text {obs }}$ sets of one star obtained at the different elevation angles. $D N_{0}$ is determined by extrapolating the best-fit line on the Langley plots to zero-airmass assuming that $\tau$ is constant during the observation. The $\tau$ of the day was calculated from the images of standard stars, and then using the same $\tau$, we cal-

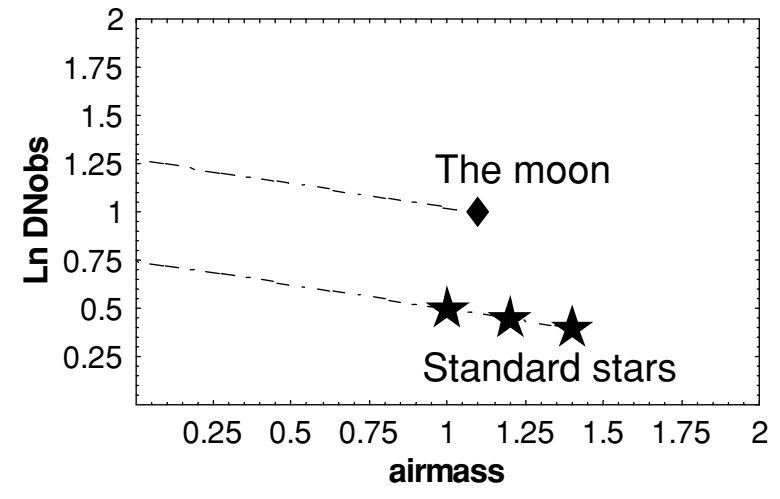

Fig. 1. An example of a Langley plot. Three observations of a standard star and one observation of the Moon are plotted. Dotted lines indicate estimated signal intensities for each airmass.

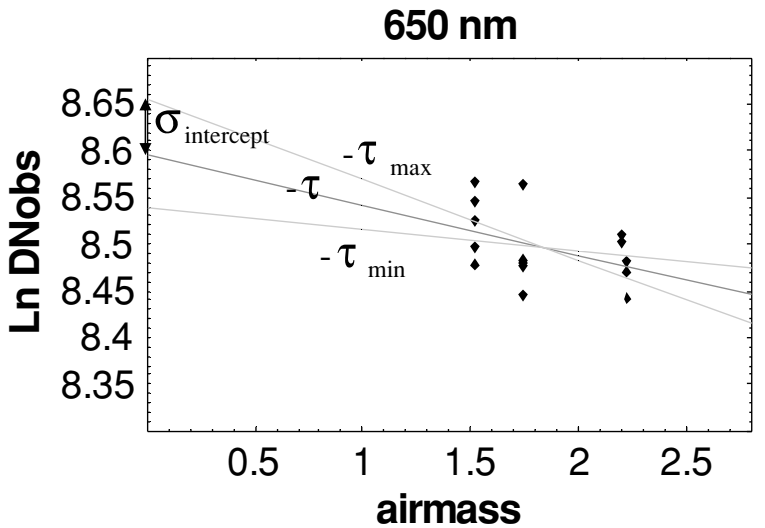

Fig. 2. A diagram showing how to define the range of the optical depth of the atmosphere, $\tau$. Solid diamonds indicate the integrated intensities of the standard star Nunki ( $\sigma$ Sagittarii) at $650 \mathrm{~nm}$ observed on 18 August 2005.

culated the extra-atmospheric intensity of the Moon from the observed intensity of the Moon. An example of how to estimate $\tau$ and its error is provided in the calculations shown in Fig. 2. The integrated intensities of the standard star Nunki ( $\sigma$ Sagittarii) observed on 18 August 2005 are plotted as solid diamonds on the Langley plot (Fig. 2). The value of $\tau$ is defined as the sign-reversed slope of the bestfit line obtained by linear regression of all data in the plot. The error of $\tau\left(\sigma_{\tau}\right)$ is determined from

$$
\begin{aligned}
\sigma_{y} & =\sqrt{\frac{1}{N-2} \sum\left(y_{i}-\left(-\tau \alpha_{i}+\ln D N_{0}\right)\right)^{2}} \\
\sigma_{\tau} & =\sigma_{y} \sqrt{\frac{\sum \alpha_{i}^{2}}{N \sum \alpha_{i}^{2}-\left(\sum \alpha_{i}\right)^{2}}}
\end{aligned}
$$


Table 2. Extra-atmospheric irradiance of Vega and the Sun at the Earth/the Moon used for this study. Gaussian transmissivity filter coefficient with 7-nm FWHM has been applied.

\begin{tabular}{llllll}
\hline & $650 \mathrm{~nm}$ & $750 \mathrm{~nm}$ & $900 \mathrm{~nm}$ & $950 \mathrm{~nm}$ & $1000 \mathrm{~nm}$ \\
\hline$I_{\text {Vega-Earth }}\left(\times 10^{-8} \mathrm{~W} / \mathrm{m}^{2} / \mu \mathrm{m}\right)$ & 2.080 & 1.373 & 0.7941 & 0.7194 & 0.6079 \\
$I_{\text {Sun-Moon }}\left(\times 10^{3} \mathrm{~W} / \mathrm{m}^{2} / \mu \mathrm{m}\right)$ & 1.548 & 1.280 & 0.9203 & 0.8210 & 0.7323 \\
$I_{\text {Sun-Earth }}{ }^{* 1}\left(\times 10^{3} \mathrm{~W} / \mathrm{m}^{2} / \mu \mathrm{m}\right)$ & 1.59 & 1.27 & 0.920 & 0.833 & 0.746 \\
$I_{\text {Sun-Earth }}{ }^{2}\left(\times 10^{3} \mathrm{~W} / \mathrm{m}^{2} / \mu \mathrm{m}\right)$ & 1.59 & 1.27 & 0.914 & 0.779 & 0.744 \\
\hline$* 1$ reference & data based on Kurucz $(1995){ }^{* 2}$ reference data based on Wehrli $(1985)$. & &
\end{tabular}

${ }^{* 1}$ reference data based on Kurucz (1995). ${ }^{* 2}$ reference data based on Wehrli (1985).

where $N$ is the number of data, and $y_{i}$ is $\ln D N_{\text {obs }}$ at airmass $\alpha_{i}$. Two light-gray lines show $\tau$ maximum, $\tau_{\max }=\tau+\sigma_{\tau}$, and $\tau$ minimum, $\tau_{\min }=\tau-\sigma_{\tau}$, respectively. The error of $\ln D N_{0}$, the $\sigma_{\text {Intercept }}$, is determined from

$$
\sigma_{\text {Intercept }}=\sigma_{y} \sqrt{\frac{N}{N \sum \alpha_{i}^{2}-\left(\sum \alpha_{i}\right)^{2}}} .
$$

The extra-atmospheric irradiance of the Moon at the Earth, $I_{\text {Moon-Earth }}$, is calculated by the following equation.

$$
I_{\text {Moon-Earth }}=I_{\text {Vega-Earth }} \times D N_{\text {Moon }} / D N_{\text {Vega }}
$$

where: $I_{\text {Vega-Earth }}$ is the extra-atmospheric irradiance of Vega at the Earth, derived from the spectral irradiance of Vega characterized by Bohlin and Gilliland (2004). The Gaussian transmissivity filter coefficient of our LCTF with 7-nm FWHM has been multiplied by the Vega irradiance spectrum. $D N_{\text {Vega }}$ and $D N_{\text {Moon }}$ is the extra-atmospheric intensity per second of Vega and the Moon calculated from observations using the Lambert-Beer law. The radiance of the Moon, $B_{\text {Moon }}$, is converted from $I_{\text {Moon-Earth }}$ as follows.

$$
B_{\text {Moon }}=I_{\text {Moon-Earth }} \times \pi \times d_{\text {Moon-Earth }}^{2} / A_{\text {pixel }}
$$

where $A_{\text {pixel }}$ is the area of lunar surface corresponding to one pixel on the lunar image calculated by (diameter of the Moon $(3476 \mathrm{~km}) /$ diameter of the Moon image (pixels) $)^{2}$, and $d_{\text {Moon-Earth }}$ is the distance between the Moon and the Earth. Reflectance of the Moon, $R_{\text {Moon }}$, is obtained by $R_{\text {Moon }}=B_{\text {Moon }} / I_{\text {Sun-Moon }} \times 100 . \quad I_{\text {Sun-Moon }}$ is the solar spectral irradiance at the Moon. To estimate $I_{\text {Sun-Moon }}$, the radiance of the Sun, $B_{\text {Sun }}$, is derived from Planck's law as follows.

$$
B_{\text {Sun }}=\frac{2 h c^{2} \pi}{\lambda^{5}\left(\exp \left(\frac{c h}{\lambda k T}\right)-1\right)}
$$

where $h$ is Planck's constant, $c$ is the speed of light, $\lambda$ is the wavelength, $k$ is the Boltzmann's constant, and $T$ is the temperature of the black body. A temperature of $5777 \mathrm{~K}$ is applied as $T$ for the solar spectrum. Solar irradiance at the Moon is calculated as follows:

$$
I_{\text {Sun-Moon }}=B_{\text {Sun }} \times G \times\left(d_{\text {Sun-Earth }} / R\right)^{2}
$$

where $G$ is the Gaussian transmissivity filter coefficient of our LCTF with 7-nm FWHM, $d_{\text {Sun-Earth }}$ is the distance between the Sun and the Earth, and $R$ is the radius of the Sun. The $I_{\text {Vega-Moon }}$ and $I_{\text {Sun-Moon }}$ used for this study are listed in Table 2 with two reference data of extraterrestrial
Table 3. Lunar data for the image at $950 \mathrm{~nm}$ on 18 August 2005.

\begin{tabular}{ll}
\hline UTC & $2005-08-18$ 9:09 \\
HST (Hawaii Standard Time) & $2005-08-18$ 23:09 \\
JST (Japan Standard Time) & $2005-08-19$ 18:09 \\
Sub-Earth_longitude & $0.819^{\circ}$ \\
Sub-Earth_latitude & $6.039^{\circ}$ \\
Sub-solar_latitude & $1.161^{\circ}$ \\
Sub-solra co-longitude & $84.114^{\circ}$ \\
Illumination & 0.9962 \\
Elevation angle & $47.2^{\circ}$ \\
Distance Earth-Moon & $3.574 \times 10^{5} \mathrm{~km}$ \\
Distance Earth-Sun & $1.012 \mathrm{AU}$ \\
Exposure time & $200 \mathrm{~ms}$ \\
Radius of the lunar image & 177 pixels \\
\hline
\end{tabular}

irradiance of the Sun; I $I_{\text {Sun-Earth }}$ is based on Kurucz (1995) and Wehrli (1985). Even if we use the reference $I_{\text {Sun-Earth }}$ instead of $I_{\text {Sun-Moon }}$ derived from Planck's law, the result will not change a lot.

The reflectance of the Moon should be normalized to match the photometric geometry of the lunar samples $(i=$ $30^{\circ}, e=0^{\circ}$ ) measured at the laboratory at Brown University (RELAB) (McEwen et al., 1998). Therefore, the irradiance of the Sun on the Moon is multiplied by $\cos 30^{\circ}$ to gain effective irradiance: $I_{\text {Sun-Moon }}^{\prime}=I_{\text {Sun-Moon }} \times \cos 30^{\circ}$. The photometric correction removes the effects of the geometry of the observing system owing to topography, phase, and libration on apparent reflectance. In this study the Clementine photometric function (Pieters et al., 1991) was applied as follows.

$$
\begin{aligned}
f_{n}= & 0.988-2.101 \times p / 100.0+2.527 \times p^{2} / 10^{4} \\
& -1.530 \times p^{3} / 10^{6}+3.367 \times p^{4} / 10^{9} \\
C_{\text {photo }}= & 0.25366 / f_{n} / \cos (i) \times(\cos (e)+\cos (i))
\end{aligned}
$$

where $p$ is the phase angle, $i$ is the incident angle, and $e$ is the emission angle. When the phase angle was less than $5^{\circ}$, we applied Yokota's function (Yokota et al., 1999) as follows.

$$
\begin{aligned}
C_{\text {photo }}= & 0.4641016 / \cos (i) \\
& \times(\cos (e)+\cos (i)) /(-0.12 \times p+2.2)
\end{aligned}
$$

Each pixel of the lunar image has a different $i$ and $e$ value, therefore $C_{\text {photo }}$ is also different. A software "LunaFilter" that makes a filter image from the lunar data, such as sub-Earth longitude, sub-Earth latitude, sub-solar latitude, sub-solar co-longitude, illumination, elevation angle, distance between the Earth and the Moon, distance between the Earth and the Sun, exposure time, and radius of lunar 

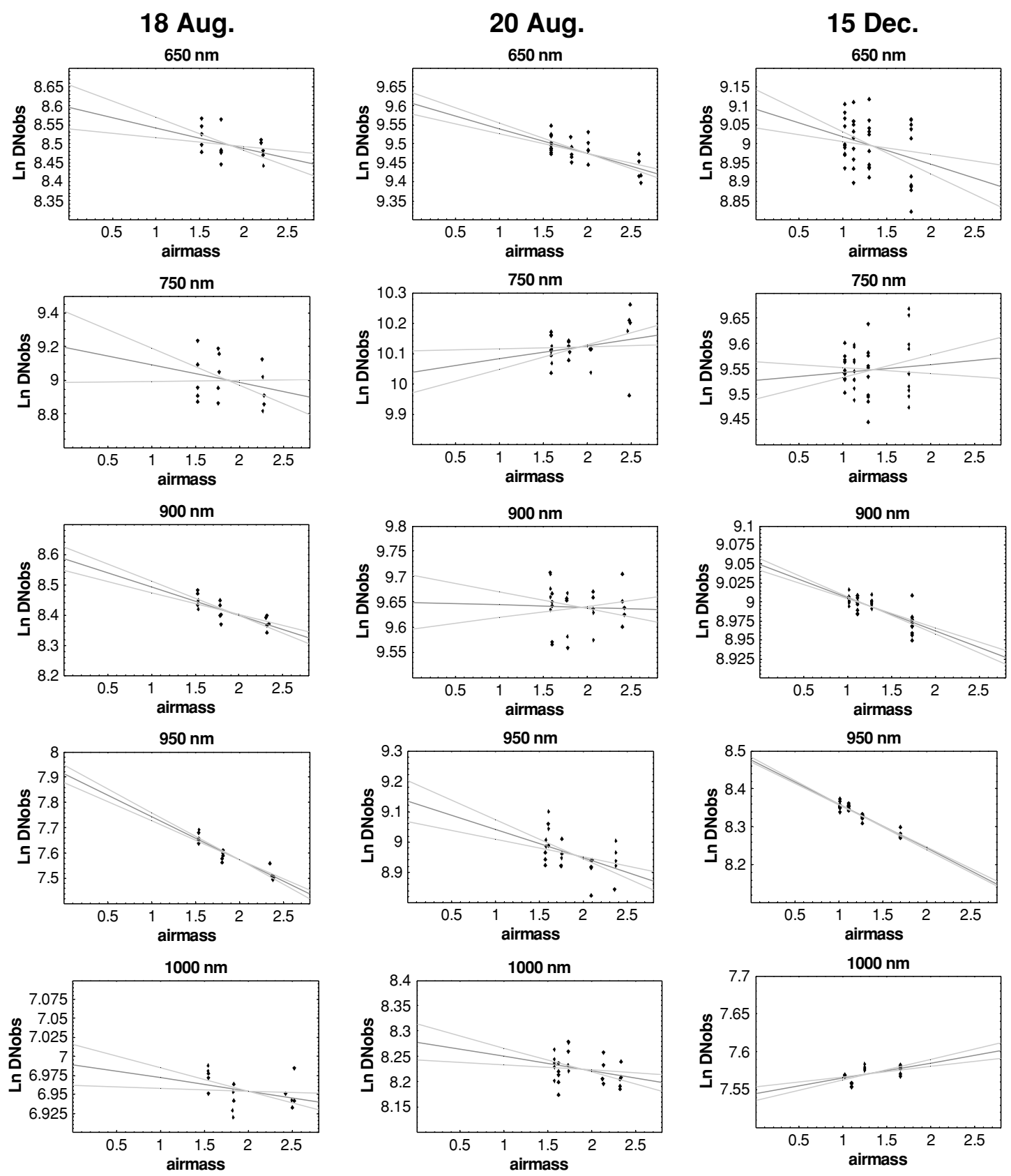

Fig. 3. The Langley plots of the standard stars at five bands on 18 and 20 August 2005 and 15 December 2005 . The center lines are the best-fit lines obtained by linear-regression of all data sets at each band. Their gradient defines $-\tau$. The lines that define $\tau_{\max }$ and $\tau_{\min }$ are also shown.

image (Table 3), has been developed and provided at the WEB site "Moon Base Osaka". Each pixel of the filter image has the value that converts the lunar image to the reflectance image. Each pixel on the filter image is calculated by the following equation.

$$
\begin{aligned}
C_{\text {filter }}= & C_{\text {photo }} \times I_{\text {Vega-Earth }} / D N_{\text {Vega }} \times d_{\text {Moon-Earth }}^{2} / A_{\text {pixel }} \\
& \times M / E / I_{\text {Sun-Moon }}^{\prime} \times \pi \times 100 / \exp (\tau(1 / \sin (\theta)))
\end{aligned}
$$

where $C_{\text {filter }}$ is the filter coefficinet (pixel value of the filter image), $E$ is the exposure time for the Moon image, $\theta$ is the altitude of the Moon, and $M$ is the coefficient used to expand the reflectance value to fit the scale of a 16 bitinteger. The resultant image has the value $D N_{\text {Moon }} \times C_{\text {filter }}=$ $R_{\text {Moon }} \times M$.

\section{Results}

Five to ten images of the standard star were obtained three to five times each night. The optical depth of the atmosphere, $\tau$, and its error, $\sigma_{\tau}$, were estimated each night from the data of the standard star. The selected standard stars and number of measurements are shown in Table 1. The atmospheric conditions during each observation were estimated using the Langley plot. The plot of the natural $\log$ of the integrated intensity of the standard star at five bands versus the airmass on 18 and 20 August 2005 and 15 December 2005 are shown in Fig. 3. The estimated optical depth of the atmosphere, $\tau$, its error, $\sigma_{\tau}$, and the R-square for the best-fit line are shown in Table 3 . In an ideal case, $\tau$ has a positive value. The fact that some $\tau$ at 750 and $1000 \mathrm{~nm}$ have a negative value indicates that the 


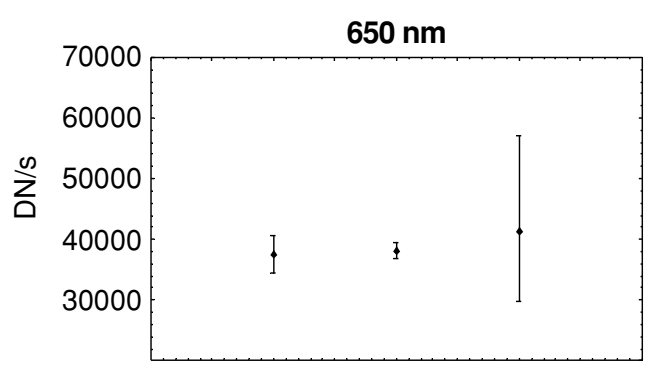

18 Aug. 20 Aug. 15 Dec. Date

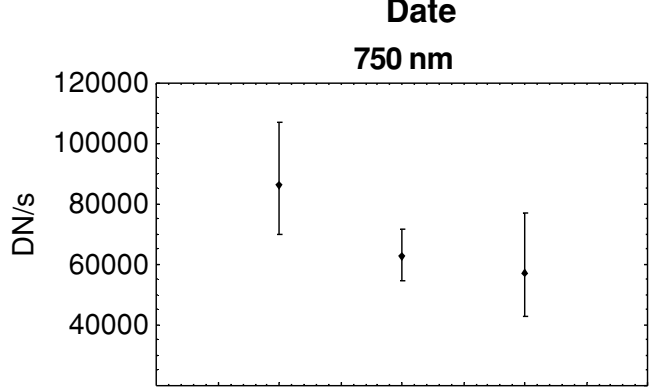

18 Aug. 20 Aug. 15 Dec. Date

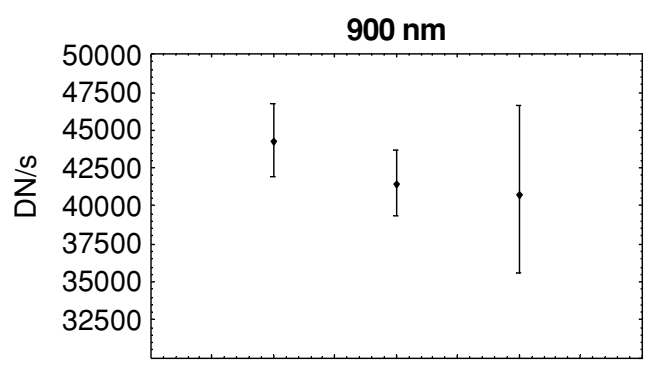

18 Aug. 20 Aug. 15 Dec. Date

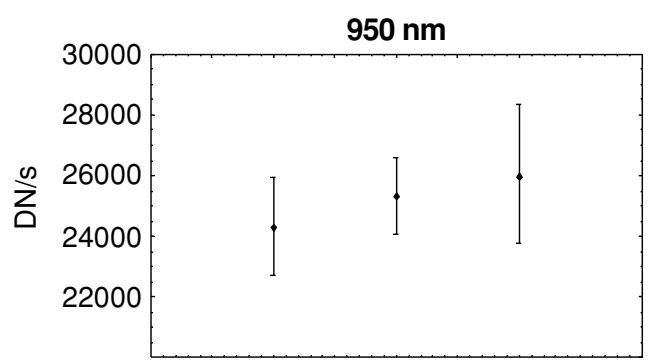

18 Aug. 20 Aug. 15 Dec. Date

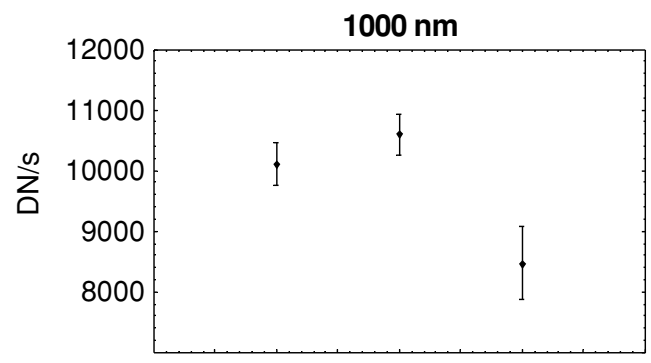

18 Aug. 20 Aug. 15 Dec. Date

Fig. 4. Estimation of the extra-atmospheric intensities of Vega and their error range. atmospheric absorptions at 750 and $1000 \mathrm{~nm}$ are unstable, probably due to water vapor.

In this study, Vega was used for the calibration standard for our telescope. The extra-atmospheric intensity $(D N)$ of Vega was calculated using the optical depth of the atmosphere, $\tau$, and Vega observations, and is shown in Fig. 4. The range of $D N_{0}$ of Vega was defined by the range of $\tau\left(\tau_{\min } \sim \tau_{\max }\right)$ and the range of $\pm 1 \sigma$ of $\ln D N_{\text {obs }}$ using the Lambert-Beer law. The data in December have a wide error range because the elevation angle of Vega was much lower then than on 18 and 20 August. The DNs of Vega show some variation, mostly owing to atmospheric conditions. If we assume that the $D N$ of Vega should be the same value within the same wavelength, Fig. 4 indicates that the data of 650,900 , and $950 \mathrm{~nm}$ were relatively insensitive to atmospheric conditions, while those of 750 and $1000 \mathrm{~nm}$ were sensitive to atmospheric conditions. This tendency is consistent with the tendency deduced from the negative value of the optical depth (Fig. 3).

The Langley-plot (Fig. 3) and R-square (Table 4) indicate that the atmospheric conditions on 18 August were the best among these three nights. The reflectance of the Moon at the five bands on 18 August was calculated, and the reflectance images are freely available to the public at the WEB site "Moon Base Osaka". Among the data obtained on 18 August, the data at $950 \mathrm{~nm}$ appears to be the most reliable because their R-square has a high value. Therefore, we will examine the lunar reflectance image on 18 August at $950 \mathrm{~nm}$ in more detail. The reflectance image and its histogram are shown in Fig. 5. The peak reflectance for the mare area is $7.2 \sim 7.4 \%$ and that for highland area is $12.8 \sim 13.0 \%$. Our lunar reflectance data were compared with Clementine UV/VIS data. Figure 6(a) is a part of our lunar reflectance image at $950 \mathrm{~nm}$. A profile of reflectance (Fig. 6 (b)) was derived along the line (Lat: $43.3^{\circ} \mathrm{S}$, Long: $20.0^{\circ} \mathrm{W}$ )-(Lat: $43.3^{\circ} \mathrm{S}$, Long: $\left.11.1^{\circ} \mathrm{W}\right)-\left(\right.$ Lat: $43.3^{\circ} \mathrm{S}$, Long: $0.0^{\circ} \mathrm{W}$ ). This line cuts across Tycho. On the other hand, Fig. 6(c) is the 950-nm reflectance image from the Clementine UV/VIS mosaic. Its profile of reflectance (Fig. 6(c)) is also derived along the line connecting the same three points. The spatial resolution and the map-projection are clearly different between these two profiles, and two lines therefore cannot perfectly coincide with each other. However, given the expectation that two lines cover almost the same area, it is obvious that the UV/VIS reflectance data are much higher than our data. The average reflectance on the line of our data is $15.2 \%$ and that of UV/VIS is $25.6 \%$. The correction factor would be approximately 0.59 . Although it is known that increasing the spatial resolution results in unexpectedly high reflectance values, it would not affect the average reflectance this much.

\section{Discussion}

Let us now estimate the error of our lunar reflectance. Among the factors expected to cause an error, the error of optical depth, $\tau$, and observed intensity, $D N_{\text {obs }}$, would be extraordinary large. Therefore, we present here the error caused by these factors as a tentative estimation. The error of extraterrestrial irradiance of the Sun, $I_{\text {Sun-Earth }}$, may be large, but it is difficult to estimate. We entrust the 
Table 4. The estimated optical depth of the atmosphere with error and coefficient of determination.

\begin{tabular}{cclllll}
\hline Date & & $650 \mathrm{~nm}$ & $750 \mathrm{~nm}$ & $900 \mathrm{~nm}$ & $950 \mathrm{~nm}$ & $1000 \mathrm{~nm}$ \\
\hline 18 Aug. 2005 & $\tau$ & 0.05420 & 0.1066 & 0.09303 & 0.1702 & 0.01739 \\
& $\sigma_{\tau}$ & 0.03194 & 0.1116 & 0.02084 & 0.0184 & 0.01361 \\
& $\mathrm{R}^{2}$ & 0.18 & 0.07 & 0.61 & 0.87 & 0.11 \\
20 Aug. 2005 & $\tau$ & 0.06576 & -0.04380 & 0.005281 & 0.09398 & 0.02874 \\
& $\sigma_{\tau}$ & 0.01446 & 0.03595 & 0.027787 & 0.03531 & 0.01851 \\
& $\mathrm{R}^{2}$ & 0.47 & 0.06 & 0.00 & 0.24 & 0.09 \\
15 Dec. 2005 & $\tau$ & 0.07249 & -0.01554 & 0.04371 & 0.1164 & -0.02032 \\
& $\sigma_{\tau}$ & 0.03783 & 0.027442 & 0.00606 & 0.0050 & 0.00697 \\
& $\mathrm{R}^{2}$ & 0.09 & 0.01 & 0.58 & 0.94 & 0.31 \\
\hline
\end{tabular}
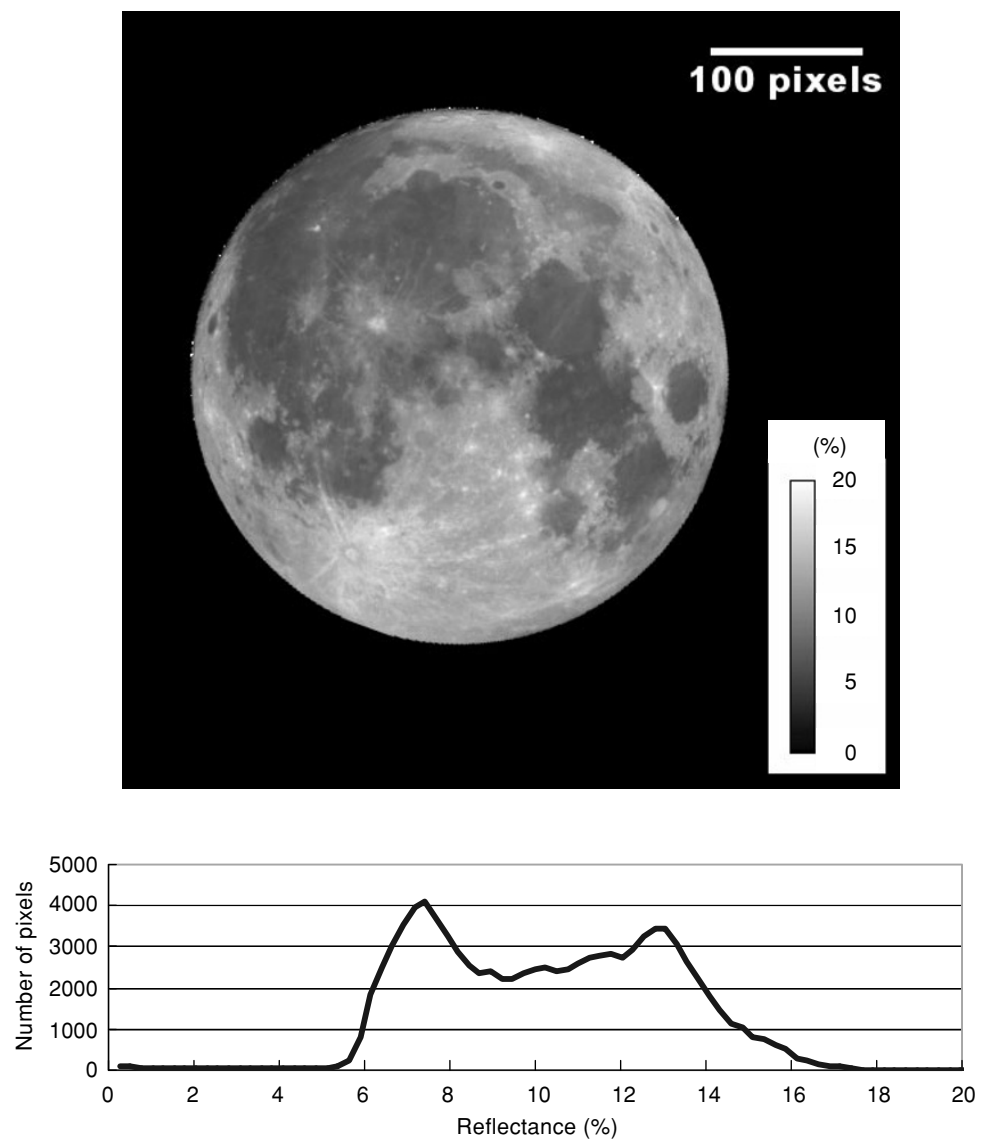

Fig. 5. The reflectance image of the Moon at $950 \mathrm{~nm}$ and its histogram.

selection of solar irradiance model and the addition of its error to the users of our reflectance data. The photometric function can also change the reflectance, and a rational estimation of its error is impossible. Therefore, we make our raw data freely available to the public and users can change the photometric function as they like. The range of $D N_{0}$ of Vega was defined by the maximum and minimum value of $D N_{0}$ as calculated by the range of $\tau\left(\tau_{\min } \sim \tau_{\max }\right)$ and the range of $\pm 1 \sigma$ of $\ln D N_{\text {obs }}$ using the Lambert-Beer law (Fig. 4). The fractional uncertainty of Vega, $\delta_{\text {Vega }}$, was determined from $\delta_{\text {Vega }}(+)=$ $\left|D N_{0}^{\text {Vega-Max }}-D N_{0}^{\text {Vega-best }}\right| / D N_{0}^{\text {Vega-best }} \times 100(\%)$, and $\delta_{\text {Vega }}(-)=\left|D N_{0}^{\text {Vega-Min }}-D N_{0}^{\text {Vega-best }}\right| / D N_{0}^{\text {Vega-best }} \times 100$ $(\%)$ (Table 5). On the other hand, it is difficult to estimate $\sigma$ of $\ln D N_{\text {obs }}$ of the Moon because of subpixel displacement of the lunar images. The integrated intensity of the lunar hemi-sphere did not change over $\pm 0.1 \%$; therefore, so long as the reflectance value is sampled not from a single pixel but from the appropriate area, the effect of scintillation would be negligible.

The range of $D N_{0}$ of the Moon was defined by the maximum and minimum value of $D N_{0}^{\text {Full-Scale }}$ calculated only by the range of $\tau\left(\tau_{\min } \sim \tau_{\max }\right)$ using the Lambert-Beer law (Fig. 4). The intensity of the brightest pixel within a lunar image was applied as $D N_{0}^{\text {Moon-Fullscale }}$. The fractional uncertainty of the Moon, $\delta_{\text {Moon }}$, was determined from $\delta_{\text {Moon }}(+)=$ $\left|D N_{0}^{\text {Moon-Max }} \quad-\quad D N_{0}^{\text {Moon-FullScale }}\right| / D N_{0}^{\text {Moon-FullScale }} \times$ $100(\%)$ and $\delta_{\text {Moon }}(-)=\mid D N_{0}^{\text {Moon-Min }}-$ $D N_{0}^{\text {Moon-FullScale }} \mid / D N_{0}^{\text {Moon-FullScale }} \quad$ \%) (Table 5). The lunar reflectance is proportional to $D N_{0}^{\text {Moon }}$ and $1 / D N_{0}^{\text {Vega }}$; therefore, the fractional uncertainty of the lunar reflectance, $\delta_{\text {Reflectance }}$, is determined from $\delta_{\text {Reflectance }}=\delta_{\text {Moon }}+\delta_{\text {Vega }}$ 


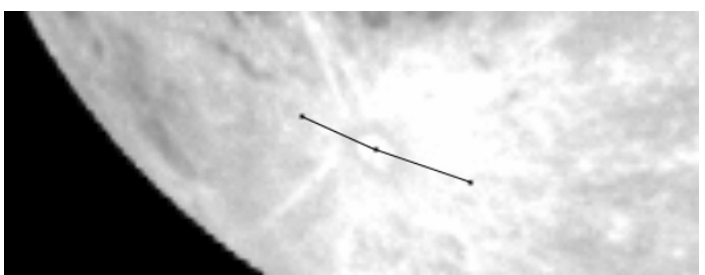

(a)

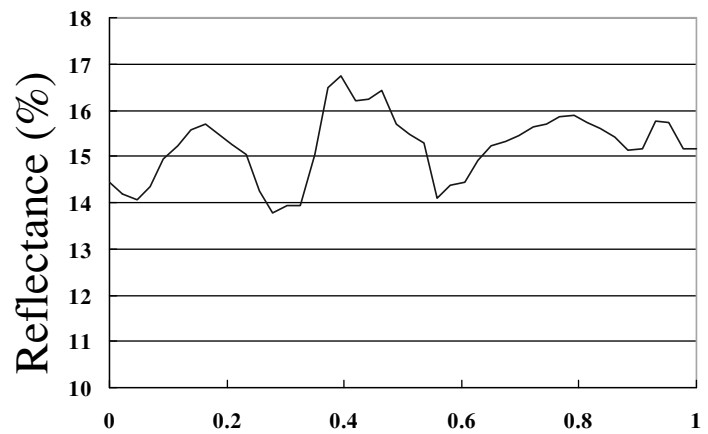

(b)

Distance

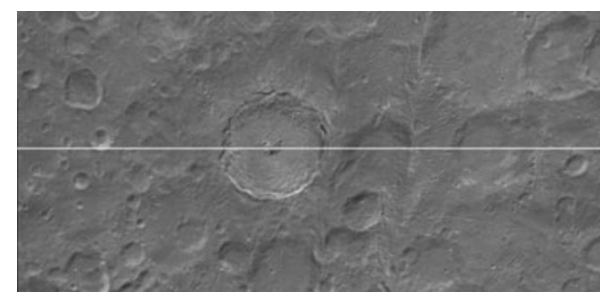

(c)

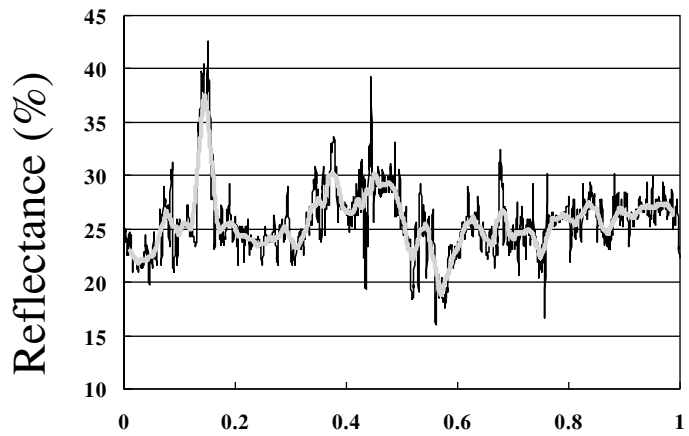

(d)

Fig. 6. Comparison between our reflectance image (a) and the Clementine UV/VIS image (c) at 950 nm around Tycho. Reflectance profiles (b) and (d) are derived from the line defined by three points: (Lat: $43.3^{\circ} \mathrm{S}$, Long: $\left.20.0^{\circ} \mathrm{W}\right)-\left(\right.$ Lat: $43.3^{\circ} \mathrm{S}$, Long: $\left.11.1^{\circ} \mathrm{W}\right)-\left(\mathrm{Lat}: 43.3^{\circ} \mathrm{S}, \mathrm{Long}\right.$ : $\left.0.0^{\circ} \mathrm{W}\right)$ on $(\mathrm{a})$ and (c), respectively. A thick line on (d) is filtered using a $10-\mathrm{km}$ moving average.

Table 5. A tentative estimation of fractional uncertainty of our results (\%).

\begin{tabular}{|c|c|c|c|c|c|c|}
\hline & & $650 \mathrm{~nm}$ & $750 \mathrm{~nm}$ & $900 \mathrm{~nm}$ & $950 \mathrm{~nm}$ & $1000 \mathrm{~nm}$ \\
\hline \multirow[t]{2}{*}{$D N_{0}$ Vega } & $\delta(+)$ & 8.31 & 23.6 & 5.69 & 6.81 & 3.48 \\
\hline & $\delta(-)$ & 7.67 & 19.1 & 5.38 & 6.38 & 3.36 \\
\hline \multirow[t]{2}{*}{$D N_{0}$ Moon } & $\delta(+)$ & 4.54 & 16.7 & 2.90 & 2.54 & 1.87 \\
\hline & $\delta(-)$ & 4.34 & 14.3 & 2.82 & 2.48 & 1.83 \\
\hline \multirow[t]{2}{*}{ Lunar reflectance } & $\delta(+)$ & 12.9 & 40.3 & 8.59 & 9.35 & 5.35 \\
\hline & $\delta(-)$ & 12.0 & 33.4 & 8.20 & 8.86 & 5.19 \\
\hline
\end{tabular}

(Table 5).

The absolute spectral calibration for Clementine reflectance data was carried out using the laboratory measurement data of lunar soil 62231 on the assumption that the reflectance spectrum of Apollo soil 62231 is representative of the calibration target area named Apollo 16 West. The discordance between Clementine data and ground-based observations (Hillier et al., 1999; Shkuratov et al., 2001; this study) indicates that this assumption should be wrong. Even if a standard soil was sampled directly from the lunar surface, the spectrum of the soil is not always representative of the area's spectra because of the heterogeneity of the lunar surface. Furthermore, 62231 had not been sampled within Apollo 16 West. The reflectance could be affected by the conditions of the measurement, such as the compaction state of the soil (M. Ohtake, personal communication). Given the estimated error (Table 5), the correction factor of the Clementine UV/VIS camera would be $0.59 \pm 0.06$ at $950 \mathrm{~nm}$. This value is very close to the value 0.532 presented by Hillier et al. (1999). When determining the absolute reflectance of the lunar surface from the lunar image obtained by the sensor of spacecrafts, ground-based observation data is more reliable as the calibration standard than laboratory measurement data of lunar soil, even though the atmosphere disturbs the ground-based observation. The accuracy of ground-based data would be improved by the further accumulation of observation data. The advantage of ground-based observations is that we can compare the obtained data with those of other scientists. Therefore, we make our data freely available to the public.

\section{Conclusion}

- The lunar reflectance images at $650,750,900,950$, and $1000 \mathrm{~nm}$ were made from the lunar images obtained with a hyper-spectral telescope located at the peak of Mt. Haleakala (Hawaii, USA).

- On the basis of a 3-day comparison of observations, the optical depths at 750 and $1000 \mathrm{~nm}$ appeared to be unstable, probably due to water vapor, and those of 650,900 , and $950 \mathrm{~nm}$ were relatively stable.

- The reflectance value of Clementine UV/VIS is much brighter than our data. The correction factor would be $0.59 \pm 0.06$ at $950 \mathrm{~nm}$. This value is close to the value 0.532 presented by Hillier et al. (1999).

The lunar reflectance images, the software tools, and the raw data are freely available to the public at the web site "Moon Base Osaka". 
Acknowledgments. We would like to express our thanks to Dr. Mike Maberry and Mr. Daniel O'Gara of Hawaii University and the ALIS (Advanced Lunar Imaging Spectrometer) users group for their kind assistance at the Haleakala site. We also wish to thank Drs. Shoichi Okano and Hiroaki Misawa of Tohoku University who allowed us to use their astronomical dome at the site and gave us helpful advice. We are grateful to Dr. David Baratoux and an anonymous reviewer for helpful and constructive suggestions. The development of the LCTF telescope and ground-based observations were supported by the Japanese Ministry of Education, Science, Sports, and Culture, Grant-in-Aid for Young Scientists (PI: K. Saiki).

\section{References}

Bohlin, R. C. and R. L. Gilliland, Hubble Space Telescope absolute spectrophotometry of Vega from the far-ultraviolet to the infrared, Astronomical J., 127, 3508-3515, 2004.

Gat, N., Imaging spectroscopy using tunable filters: a review, Proc. SPIE, 4056, 50-64, 2003.

Hillier, J. K., B. J. Buratti, and K. Hill, Multispectral photometry of the Moon and absolute calibration of the Clementine UV/Vis camera, Icarus, 141, 205-225, 1999.

Kieffer, H. H. and T. C. Stone, The spectral irradiance of the Moon, Astronomical J., 129, 2887-2901, 2005.

Kurucz, R. L., The solar irradiance by computation, Proc. of the 17th Annual Review Conference on Atmospheric Transmission Models, Geophysics Directorate/Phillips Laboratory, 333-334, 1995.
Langley, S. P., The bolometer and radiant energy, Proc. Am. Acad. Arts Sci., 8, 343-358, 1881.

McEwen, A. S., A precise lunar photometric function, LPSXXVII, 841$842,1996$.

McEwen, A. S., E. Eliason, P. Lucey, E. Malaret, C. Pieters, M. Robinson, and T. Sucharski, Summary of radiometric calibration and photometric normalization steps for the Clementine UVVIS images, LPSCXXIX, 1998

Pieters, C., S. Pratt, H. Hoffmann, P. Helfenstein, and J. Mustard, Bidirectional spectroscopy of returned lunar soils: detailed "ground truth" for planetary remote sensors, LPSCXXII, 1069-1070, 1991.

Shiobara, M., J. D. Spinhirne, A. Uchiyama, and S. Asano, Optical depth measurements of aerosol, cloud, and water vapor using Sun photometers during FIRE Cirrus IFO II., J. Appl. Meteor., 35, 36-46, 1996.

Shkuratov, Y. G., V. G. Kaidash, M. A. Kreslavsky, and N. V. Opanasenko, Absolute calibration of the Clementine UVVIS data: comparison with ground-based observation of the Moon, Solar System Res., 35, 29-34, 2001.

Wehrli, C., Extraterrestrial solar spectrum, Pub. 615, PhysikalischMeteorologisches Observatorium and World Radiation Center, pp. 23, 1985.

Yokota, Y., R. Iijima, R. Honda, T. Okada, and H. Mizutani, Photometric properties of the Moon: phase curves at small phase angles $\left(0-10^{\circ}\right)$ by Clementine images, Adv. Space Res., 23, 1841-1844, 1999.

K. Saiki (e-mail: ksaiki@ess.sci.osaka-u.ac.jp), K. Saito, H. Okuno, A. Suzuki, Y. Yamanoi, N. Hirata, and R. Nakamura 